\title{
La cohésion lexicale des noms sans déterminant après il $y$ a et dans la phrase nominale
}

\author{
Yumi Takagaki* \\ Université Kwansei Gakuin, Japon ; Laboratoire Ligérien de Linguistique
}

\begin{abstract}
Résumé. L'objectif de cette étude est de mettre en lumière de quelle manière la cohésion peut être réalisée par des moyens lexicaux dans les deux constructions suivantes : il y $a+$ nom nu et la phrase nominale dont la tête est un nom nu. Malgré l'absence de déterminant, ces noms gardent leur nature substantive. L'analyse quantitative révèle quelques différences entre les noms inclus dans ces deux constructions, dont notamment la présence d'un marqueur de prédication plus fréquente devant la phrase nominale et la plus grande variété des noms nus les plus fréquemment employés après $i l$ y $a$ par rapport à ceux des phrases nominales. De plus, avec l'identification des usages typiques de chaque construction, pour il y $a+$ nom nu [Définition/ Reconnaissance d'une existence/Présentation d'un événement] et pour la phrase nominale à nom nu [Reformulation/Présentation d'un concept/ Jugement], l'examen de l'orientation informationnelle du nom nu permet d'établir une distinction, concernant la cohésion, entre les deux constructions. Dans la phrase nominale, avec un nom nu et en dehors de tout recours à des procédés syntaxiques, prévaut une perspective rétrospective.
\end{abstract}

\begin{abstract}
Lexical cohesion of bare nouns after "il $y a$ " and in nominal sentences whose head is a bare noun. This study aims to clarify how cohesion can be achieved through lexical means in the two constructions: "il y $a+$ bare noun" and nominal sentences whose head is a bare noun. Despite the absence of a determiner, these nouns retain their substantive nature. Our quantitative analysis revealed some differences between the nouns included in these two constructions. Particularly, there were more predication markers in front of the nominal sentence whose head is a bare noun. Moreover, there was a wider variety among the most frequently used bare nouns after "il y a," compared to nominal sentences whose head is a bare noun. Furthermore, having identified the typical uses of each construction (for "il y a + bare noun": definition, recognition of an existence or presentation of an event; and for nominal sentences whose head is a bare noun: reformulation, presentation of a concept or judgment), the examination of the informational orientation of bare nouns allowed us to distinguish the two constructions in terms of cohesion. The nominal sentence, which introduces a bare noun without syntactic procedures always has retrospective informational orientation.
\end{abstract}

\footnotetext{
*yumi.takagaki@kwansei.ac.jp
} 


\section{Introduction}

L'objectif de cette étude est d'examiner de quelle manière la cohésion peut être réalisée par des moyens lexicaux. Dans ce but, nous examinerons les noms dépourvus de déterminant (désormais $ø \mathrm{~N}$ ) dans les deux constructions suivantes : il y $a ø N$ et la phrase nominale dont la tête est un $\varnothing \mathrm{N}$. La première introduit un $\varnothing \mathrm{N}$ avec une tournure spéciale, ce qu'on appelle "présentatif». La seconde introduit dans le texte un nom sans recours à des procédés syntaxiques.

En voici deux exemples avec le même nom guerre. (Dans tous les exemples, c'est nous qui mettons en gras.)

(1) “Aux sources de la guerre économique”, d'Ali Laïdi (Armand Colin)

"La guerre économique est partout", écrit Ali Laïdi. "Il y a guerre lorsqu'une entreprise ou un Etat use de moyens déloyaux ou illégaux pour atteindre son objectif."

(Philippe Arnaud, Le Monde, en chapeau de son article du 3/12/2012)

(2) Mais tandis que l'Europe prend ainsi le chemin de la paix, c'est la guerre qui sévit en Asie du sud-est. Guerre injuste car elle résulte en fait de l'intervention armée des États-Unis sur le territoire du Vietnam, guerre détestable puisqu'elle conduit une grande nation à en ravager une petite.

(Discours du général De Gaulle, à l'occasion de la fin de l'année 1966)

$\mathrm{Si}$ ces deux constructions partagent plusieurs caractéristiques, il existe quelques différences importantes. En effet, si dans certains cas elles sont interchangeables ${ }^{1}$, ce n'est pas le cas de (1) et (2). Cette différence nous incite à examiner ces deux constructions afin de mettre en lumière le mécanisme d'une cohésion exclusivement lexicale ainsi que les conditions qui la décident.

Dans ce qui suit, la section 2 définira l'absence de déterminant et la portée de la présente étude. La section 3 montrera que les noms nus que nous traitons gardent leur nature substantive. La section 4 présentera une analyse quantitative permettant de saisir les tendances générales de la nature de ces noms nus. La section 5 identifiera les usages types de chaque construction, lesquels révéleront une différence de cohésion entre il y $a$ et la phrase nominale.

\section{Méthode et données}

\subsection{Une définition de l'absence de déterminant}

Selon Riegel, Pellat et Rioul (2018), le déterminant se définit comme «le mot qui doit nécessairement précéder un nom commun pour constituer un groupe nominal bien formé. » Or, les exemples (1) et (2) montrent qu'un nom sans déterminant peut être bien formé sous certaines conditions. Si ces auteurs énumèrent une série de cas présentant une absence de déterminant, ils ne précisent pas vraiment ce qui garantit leur validité grammaticale.

Nous désignerons comme nom nu (ø $N)$ un nom dépourvu de déterminant lexicalement réalisé, qu'il s'agisse d'articles (le, un, des, $d u \ldots$..), de déterminants possessifs (mon, ton, son...), de démonstratifs (ce), d'interro-exclamatifs (quel) ou d'un déterminant dit indéfini comme certain, chaque, plusieurs... L'important est le fait que ces déterminants ne sont pas cumulables. Ce caractère non cumulable exclut les expressions comme autre, divers, tout qui peuvent être employées avec un article défini ou indéfini (e.g. un autre fait, les diverses questions, toutes les choses). 
Si un nom nu est accompagné d'un ou plusieurs qualificatifs ou «marqueurs de prédication » tels que mais, pas et puis au sens de Lefeuvre (1999b) cité infra, la désignation $n u(\varnothing)$ sera conservée en considérant que l'absence de déterminant est prépondérante.

\subsection{La portée de l'étude}

Notre recherche s'appuie sur des attestations écrites, y compris éventuellement de l'écrit oralisé, comme en (2). Les textes s'inscrivent dans l'écrit journalistique, scientifique et littéraire.

Nous limitant aux données pertinentes à l'observation de la cohésion lexicale, nous excluons certaines tournures, notamment les « expressions lexicalisées » (cf. Picabia 1987). D'abord les locutions figées telles que la locution il y a anguille sous roche qui forme un tout indissociable pour la compréhension du sens. Cependant le degré de figement est souvent difficile à déterminer. On retiendra comme critère l'impossibilité d'insertion d'un déterminant. À ce titre, nous considérons que sont des locutions figées il y a lieu de s'inquiéter / Attention! qui n'acceptent pas l'interpolation d'un déterminant.

Seront également exclues les constructions qui, syntaxiquement, rendent superflu le déterminant, par exemple en cas de coordination de la forme $N_{1}$ et $N_{2}$ telle que thé et café ou dans une énumération comme chiens, chats, poissons, oiseaux, cochons d'Inde et poules. Certaines variétés de il y a requièrent syntaxiquement un nom nu : il y a ø $N_{i}$ et ø $N_{i}$ quand les deux N sont identiques comme dans Il y a cahier et cahier (Philippe Lejeune, Signes de vie, le pacte autobiographique, 2005) et si ø $N$, il y a comme dans Si leçon il y a, elle se passe devant le miroir (Antoine Blondin, Ma vie entre les lignes, 1982).

Quant aux noms en position frontale, seules sont concernées les phrases averbales. Un syntagme averbal faisant partie d'une structure verbale sera donc exclu. Dans la phrase Héritier d'une autre époque, Juan Antonio Samaranch ne peut être l'homme qui mènera une telle révolution. (Le Monde, 26/01/999), le nom nu Héritier est apposé au sujet qui est suivi du prédicat verbal. Cette phrase n'est pas nominale.

\section{Le caractère substantif de $\varnothing \mathbf{N}$}

Les noms nus considérés gardent leur nature substantive. La preuve en est la reprise par un pronom, l'insertion d'un article et la forme plurielle.

Commençons par il y a. En (3), le ø $\mathrm{N}$ blocage est repris par le (dans tous les exemples, le soulignement est le nôtre) :

(3) Pendant dix bonnes minutes, Joxe continue sur ce ton désagréable pour Edith Cresson, avec une passion telle que même Jack Lang délaisse un instant sa principale activité dans les Conseils des ministres : faire son courrier.

C'est Mitterrand soi-même qui devra apaiser tout le monde en lançant: "Qu'on le veuille ou non, il y a blocage et il faudra le résoudre. "

(Canard enchaîné, 27/09/1989)

La reprise par le pronom neutre en est également possible, comme illustré en (4).

(4) Tout d'abord sous la forme de la neutralisation : en français la modalité subjonctif ou la préposition de appartiennent à des ensembles oppositionnels (subjonctifindicatif, de-à-autres prépositions). Mais ils peuvent devenir des «cartes forcées » : ainsi dans je crains qu'il ne soit ou cela provient de. En stricte rigueur on devrait donc considérer le même phénomène comme pertinent lorsqu'il $\mathbf{y}$ a opposition, comme non pertinent lorsqu'il n'y en a pas. 
(Frédéric François et al. La syntaxe de l'enfant avant 5 ans, 1977)

En (4) l'insertion de une après il y a accroîtrait la précision de la phrase -s'il s'agissait, par exemple, d'une opposition entre le Président des États-Unis et le Président russe.

Dans l'exemple (5), qui contient plusieurs il y a ø $N$, deux groupes nominaux dépourvus de déterminants (perversion sexuelle et écoute téléphonique) sont repris par le pronom personnel sujet elle.

(5) II y a mort d'homme ; mais la mitraillette ne crépite que huit pages avant la fin. Il y a perversion sexuelle ; mais elle est seulement évoquée, jamais décrite. Il y a écoute téléphonique ; mais elle demande moins d'effort que nous n'en dépensons à réparer un aspirateur. Il y a filature; mais les gabardines n'y sont point de rigueur, le bord du chapeau pas obligatoirement rabattu sur les yeux du tueur, et le chewing-gum est tout à fait absent. Enfin, et surtout, il y a services secrets ;

(Pierre Ajame, Le Nouvel Observateur, 24-30/09/1979)

Dans la première phrase II y a mort d'homme, l'insertion d'un article indéfini devant mort est possible. Sans article, la construction présente l'événement, sans livrer d'information sur le nombre de morts.

Dans la phrase nominale, la pronominalisation est également possible, comme Guerre en (2) et Conclusions, au pluriel, en (6). Ce caractère pluriel indique que le nom n'a pas de valeur attributive.

(2') Mais tandis que l'Europe prend ainsi le chemin de la paix, c'est la guerre qui sévit en Asie du sud-est. Guerre injuste car elle résulte en fait de l'intervention armée des États-Unis sur le territoire du Vietnam, guerre détestable puisqu'elle conduit une grande nation à en ravager une petite.

(6) Nous avions déjà l'art d'opinion, la démocratie d'opinion ; nous aurons maintenant l'enseignement d'opinion. Il faut renoncer à l'idée que le lycée puisse rester un lieu privilégié de communication du savoir.

Conclusions? Elles sont au nombre de deux. La première était déjà contenue dans mes prémisses.

(Le Nouvel Observateur, 22/10/1998, cité par Naganuma 2004)

Leur valeur référentielle témoigne de la nature substantive de ces noms nus.

La pronominalisation, la possibilité d'insérer un déterminant et la pluralité contreviennent à une valeur attributive et confirment la nature substantive des noms.

\section{Le sens des noms nus}

Dans cette section, on examinera quels sont les types de noms nus les plus fréquemment utilisés dans les deux constructions. Les données sont collectées à partir des 1360 textes publiés entre 1950 et aujourd'hui mis en ligne sur Frantext.

\subsection{Les noms nus les plus employés après il y a}

On compte 3984 noms nus après il y a. Le tableau 1 liste les 90 noms ayant 5 occurrences ou plus. Ils totalisent 1479 occurrences soit $37 \%$ de l'ensemble. 
Tableau 1. Les noms nus les plus fréquemment utilisés après il y $a$.

\begin{tabular}{|l|l|}
\hline nombre d'occurrences & noms \\
\hline 202 & moyen \\
\hline 106 & besoin \\
\hline 97 & place \\
\hline 80 & temps \\
\hline 75 & foule \\
\hline 43 & trace \\
\hline 40 & urgence \\
\hline 37 & erreur \\
\hline 36 & avantage \\
\hline 32 & danger \\
\hline 28 & matière \\
\hline 21 & rupture \\
\hline 18 & contradiction, fête \\
\hline 16 & conflit, prescription \\
\hline 14 & bal, continuité, possibilité, \\
\hline 13 & mouvement, opposition, plaisir, risque \\
\hline 12 & accord, connexion, incompatibilité, répétition \\
\hline 11 & changement, translation \\
\hline 10 & doute, péril, tendance \\
\hline 9 & apparence, cö̈ncidence, équivalence, identité \\
\hline 8 & affluence, âme, concurrence, faute, pénurie \\
\hline 7 & $\begin{array}{l}\text { abondance, augmentation, confusion, conseil, contact, formation, } \\
\text { grève, hésitation, progrès }\end{array}$ \\
\hline 6 & $\begin{array}{l}\text { absence, baisse, communication, conscience, correspondance, } \\
\text { école, égalité, entente, excès, obligation, séparation, sujet }\end{array}$ \\
\hline 5 & $\begin{array}{l}\text { corrélation, discordance, espoir, histoire, passage, perte, réunion, } \\
\text { violation }\end{array}$ \\
\hline $\begin{array}{l}\text { apparition, bataille, cinéma, composition, concomitance, création, } \\
\text { crime, dédoublement, divergence, échange, émission, liberté, } \\
\text { maldonne, menace, musique, présentation, recours, représentation, } \\
\text { solidarité, volonté }\end{array}$ \\
\hline
\end{tabular}

Les plus fréquents sont souvent des noms qui peuvent être combinés avec un infinitif à l'aide d'une préposition: besoin / moyen de inf; place pour inf; avantage / danger / urgence à inf.

Ces noms sont majoritairement abstraits et beaucoup expriment un événement (bal, bataille, crime, fête, grève, réunion), un phénomène (conflit, menace, répétition, rupture) ou une relation (accord, coüncidence, concomitance, connexion, continuité, contradiction, corrélation, correspondance, discordance, égalité, équivalence, identité, incompatibilité, opposition).

Ajoutons à ce tableau un autre résultat quantitatif: les adjectifs qualifiant les $\varnothing \mathrm{N}$ manquent de variété : parmi 411 noms nus précédés d'un adjectif, on trouve 196 occurrences de autre chose, 71 de beau temps et 37 de grand + nom.

\subsection{Les noms nus les plus employés dans les phrases nominales}

On dénombre 1469 noms nus en phrase nominale. Le tableau 2 inventorie les noms présentant 5 occurrences ou plus, soit 20 types pour 841 occurrences, soit $57 \%$ du nombre total. 
Tableau 2. Les noms nus les plus fréquemment utilisés dans la phrase nominale.

\begin{tabular}{|l|l|}
\hline nombre d'occurrences & noms \\
\hline 400 & question \\
\hline 182 & besoin \\
\hline 86 & moyen \\
\hline 37 & envie \\
\hline 27 & trace \\
\hline 12 & visite \\
\hline 11 & silence \\
\hline 9 & faim, retour \\
\hline 8 & confiance, patience \\
\hline 7 & âme, malheur, peur \\
\hline 6 & courage \\
\hline 5 & séance, touche, travail, trêve, trou \\
\hline
\end{tabular}

Ce qui n'apparaît, c'est que la plupart de ces noms sont précédés d'un " marqueur de prédication » (Lefeuvre 1999b), tel que pas, puis, mais. Ainsi, 393 occurrences sur 400 de question sont précédées du marqueur de négation pas; toutes les occurrences de besoin, de moyen, de envie et de trace également. 11 occurrences sur 12 de visite sont précédées de puis et une de mais. Pour silence, 9 sont employés avec puis et 2 avec mais. Quant à retour, sur 9 occurrences, 8 ont puis en cooccurrence et, pour confiance, on en compte 6 avec pas et 2 avec mais.

\subsection{Différences entre les noms nus employés dans les deux constructions}

Les noms présents dans les deux tableaux permettent de saisir quelques tendances générales. En comparant les listes, on se propose de faire ressortir les ressemblances et les différences entre les deux constructions.

D'abord on a constaté la fréquence très élevée des marqueurs de prédication pas, puis, mais dans le cas d'une phrase nominale à $\varnothing \mathrm{N}$; ils semblent indispensables pour certaines d'entre elles. À l'inverse, ils sont plus rares pour il y a ø $N$. Cette différence illustre la façon dont les deux constructions produisent de la cohésion. Comme le dit Lefeuvre (1999b), ces marqueurs de prédication ont pour fonction d'indiquer que tel terme averbal remplit un rôle prédicatif; sinon, un groupe substantival serait difficilement interprété comme un groupe prédicatif. Par ailleurs, un tel risque n'existant pas pour la construction il y a ø $\mathrm{N}$, elle peut très bien s'en passer.

Ensuite, la sélection lexicale diffère. La variété de noms nus les plus fréquemment employés après il y a est plus importante que ceux employés dans les phrases nominales. Statistiquement, le tableau 1 comprend 90 noms contre 20 pour le tableau 2. L'application du ratio type-occurrence, qui mesure la diversité lexicale, donne 0,0609 pour il y a ø $N$ et 0,0238 pour la phrase nominale à $ø \mathrm{~N}$.

Or, l'examen des exemples dont les occurrences sont inférieures à 5 - elles ne figurent pas dans les tableaux ci-dessus - révèle un autre aspect qui distingue les deux constructions. Si les noms cités sont pour la plupart abstraits, il existe quelques exemples avec des noms nus concrets et ceux-ci se rencontrent majoritairement dans les phrases nominales. Il semble que il y $a$ accepte très difficilement des noms nus concrets, à moins qu'ils ne soient interprétés comme des événements. ( $c f$. 5.1.3.) De plus, par rapport à la phrase nominale, $i l$ y $a$ semble difficilement accepter un nom nu au pluriel.

À la différence du $\varnothing N$ dans il y $a$, le nom nu en position frontale d'une phrase nominale ne semble pas contraint lexicalement. S'il est le plus souvent abstrait et singulier, le concret (cf. infra, Couvercle en (14)) et le pluriel (cf. supra, Conclusions en (6)) sont tout à fait possibles. 


\section{Trois usages de il y a o $\mathbf{N}$}

Dans cette section, on examinera quelques caractéristiques qui échappent à l'analyse quantitative. Dans ce but, on identifiera des usages typiques de chaque construction dont le fonctionnement textuel diffère.

\subsection{Trois usages de il y a ø $\mathrm{N}$}

La construction il y a a plusieurs emplois : certaines sont interchangeables avec il existe et d'autres non. Selon l'interprétation, on identifie trois emplois de il y a ø $N$.

Type I : Définition

Type II : Reconnaissance d'une existence

Type III : Présentation d'un événement ${ }^{2}$

Deux critères les distinguent: l'information nouvelle/ancienne et l'orientation informationnelle.

\subsubsection{Type I : Définition}

Le premier cas de figure de il y a ø $N$ concerne un contexte de définition. Dans ce cas, très souvent, le nom nu après il y $a$ se trouve dans le cotexte antérieur, comme illustré en (7).

(7) Nous commencerons par distinguer deux types de relations causales: la relation causale au sens fort et la relation causale au sens faible. En voici les définitions :

relation causale (sens fort)

Il y a relation causale au sens fort lorsque l'opérateur CAUSE a pour argument un événement et un état ou un événement, l'événement causant directement l'état ou l'événement.

relation causale (sens faible)

Il y a relation causale au sens faible lorsque l'opérateur CAUSE a pour argument deux éventualités, états et/ou événements, discontinues dans le temps. (Jacques Moeschler «L'expression de la causalité en français », 2003)

Pour mettre en évidence la structure informationnelle qui spécifie ce type par rapport aux deux suivants, nous nous référons à Prince (1981, 1992), qui distingue (i) le caractère nouveau/ancien de l'information du point de vue du discours (discourse-new/old) et (ii) les connaissances supposées du/des destinataire(s) (hearer-new/old). En référence à cette taxonomie, Togo (2011) soutient que la construction il y a sert à introduire un thème supposé connu du/des destinataire(s) (hearer-old) mais discursivement nouveau (discourse$n e w)$. S'il retient cette distinction pour expliquer les apparitions des déterminants définis après il y $a$, il n'aborde pas la question des déterminants zéro. Son observation nous semble pourtant pertinente, du moins partiellement, pour rendre compte du type il y a ø $N$.

En (7), le terme dépourvu de déterminant relation causale employé après il y a apparaît déjà dans le cotexte antérieur. Il est donc connu (hearer-old). Mais du point de vue discursif, il a un caractère nouveau d'information (discourse-new); c'est avec il y a qu'on inaugure les conditions de l'utilisation de ce terme. il y a ø $N$ s'emploie souvent ainsi comme phrase définitoire.

Même s'il ne s'agit pas, strictement parlant, de cas de définition, il y a ø $N$ est souvent employé lorsqu'on présente les conditions d'apparition d'un phénomène. C'est le cas de (8), qui contient une itération d'économie. 
(8) La tendance contraire à l'amplification est l'économie, qui se traduit par un resserrement de l'énoncé obtenu par la rédaction, en nombre ou en étendue, des signes qui le composent. Il y a économie dans un segment de l'énoncé lorsque le même signifié est porté par un signifiant allégé.

(Jean-Paul Vinay et Jean Darbelnet, Stylistique comparée du français et de l'anglais, 1966)

Il en va de même pour (1) : la répétition de guerre et la construction avec il y a précisent les conditions de son déclenchement.

(1') “Aux sources de la guerre économique”, d'Ali Laïdi (Armand Colin)

"La guerre économique est partout", écrit Ali Laïdi. "Il y a guerre lorsqu'une entreprise ou un Etat use de moyens déloyaux ou illégaux pour atteindre son objectif."

Ce premier type définitoire de il y a ø $N$ caractérisé par la répétition se différencie des deux types suivants par une structure informationnelle où $\varnothing \mathrm{N}$, supposé connu du/des destinataire(s) (hearer-old), est discursivement nouveau (discourse-new).

\subsubsection{Type II : Reconnaissance d'une existence}

L'environnement qui légitime l'apparition de il y a øN ne se limite pas au cas de la définition. En (9), le nom nu employé juste après il y a apparaît pour la première fois dans le texte. L'information véhiculée par pléonasme est nouvelle à la fois du point de vue du discours et du point de vue des connaissances partagées (discourse new/hearer new selon la taxonomie de Prince), puisque l'auteur constate pour la première fois l'existence d'un phénomène que le lecteur ne connaît pas.

(9) L'essentiel est dit: à quoi bon s'étendre sur les informations superflues que donneraient le dessin et le modelé, d'une manière plus théorique et moins évocatrice ? Il y aurait pléonasme. Les déterminations foudroyantes auxquelles procède son œil vont parfois jusqu'au défi, en prenant le contre-pied de notre attente.

(René Huyghe, Dialogue avec le visible, 1955)

Dans ce texte, après avoir décelé un phénomène, au moyen de la construction il y a ø $N$, le critique livre son jugement sur ce qu'il désigne comme un pléonasme. Il s'agit à la fois d'un constat et d'une prédication extraphrastique. Le $\varnothing \mathrm{N}$ commente rétrospectivement le cotexte antérieur.

Pour décrire l'orientation informationnelle, on reprend le regard prospectif/rétrospectif, une distinction élaborée par Lefeuvre (2005). D'après elle, le regard prospectif s'établit lorsqu'il intervient avant que l'information ne soit explicitée. À l'inverse, le regard sera rétrospectif, après que l'information a été donnée. Ces deux fonctions peuvent se cumuler dans certains énoncés, à la fois prospectifs et rétrospectifs. Il existe donc trois cas selon que le prédicat joue un rôle uniquement prospectif, uniquement rétrospectif ou à la fois prospectif et rétrospectif. Dans il y a ø $N$ du Type I du 5.1.1, le contexte de définition requiert un regard à la fois prospectif et rétrospectif : « prospectif » pour la partie qui définit le terme et « rétrospectif » pour le nom répété.

Quant au Type II, le ø N est rétrospectif comme en (3) qui entre dans le Type II. Le ø N blocage est rétrospectif en ce sens qu'il fonctionne comme conceptualisation de ce qui a été décrit antérieurement dans le cotexte. 
(3') Pendant dix bonnes minutes, Joxe continue sur ce ton désagréable pour Edith Cresson, avec une passion telle que même Jack Lang délaisse un instant sa principale activité dans les Conseils des ministres : faire son courrier.

C'est Mitterrand soi-même qui devra apaiser tout le monde en lançant: «Qu'on le veuille ou non, il y a blocage et il faudra le résoudre. »

\subsubsection{Type III : Présentation d'un événement}

Ce Type III ressemble au Type II en ce qui concerne la nouveauté informationnelle. La différence réside dans l'orientation du regard qui est prospectif. La construction ne conceptualise pas le contenu du cotexte précédent mais elle présente un événement nouveau. Les exemples sont nombreux dans nos données : il y a fête / bal / grève / rupture / conflit / répétition. Le Type III semble le plus fréquent dans les occurrences de cette tournure. Se classent dans cette rubrique il y a ø $N$ en (4) et les cinq occurrences de la construction en (5) reproduits ci-dessous.

(4') Tout d'abord sous la forme de la neutralisation : en français la modalité subjonctif ou la préposition de appartiennent à des ensembles oppositionnels (subjonctifindicatif, de-à-autres prépositions). Mais ils peuvent devenir des «cartes forcées » : ainsi dans je crains qu'il ne soit ou cela provient de. En stricte rigueur on devrait donc considérer le même phénomène comme pertinent lorsqu'il y a opposition, comme non pertinent lorsqu'il n'y en a pas.

(5') II y a mort d'homme; mais la mitraillette ne crépite que huit pages avant la fin. Il y a perversion sexuelle ; mais elle est seulement évoquée, jamais décrite. Il y a écoute téléphonique ; mais elle demande moins d'effort que nous n'en dépensons à réparer un aspirateur. Il $\mathbf{y}$ a filature; mais les gabardines n'y sont point de rigueur, le bord du chapeau pas obligatoirement rabattu sur les yeux du tueur, et le chewing-gum est tout à fait absent. Enfin, et surtout, il y a services secrets ;

Ces noms nus exprimant un événement ou un phénomène sont normalement abstraits ; ceux qui sont à première vue concrets ne désignent pas d'objets tangibles. En (10), école ne signifie pas le lieu où l'on dispense un enseignement, mais la formation.

(10) Arrêtez la marelle et couchez-vous vite! Demain il y a école pour les petites filles!

(Jean Anouilh, Chers zoiseaux, 1977)

Il en va de même en (11) où homme désigne non pas un être vivant spécifique mais l'espèce humaine en général.

(11) ils ne voyaient personne des jours d'affilée, les seules nouvelles leur venaient des chauffeurs de la Rollbahn qui remontaient la ligne ; tout autour d'eux, plate et vide, s'étalait la vaste terre : $Y$ a-t-il homme qui vive en cette plaine? chante le preux du conte russe.

(Jonathan Littell, Les Bienveillantes, 2006)

En (12), la phrase il y a atteste la non-existence d'un phénomène, lequel n'a pas besoin d'être déterminé et encore moins référé. Dans la négative, le référent du nom n'est pas présupposé ce qui rend possible l'absence de déterminant.

(12) Comment aurais-je pu haïr Apolline qui m'aime plus qu'elle-même ? Comment aurais-je pu ne pas désirer celle où tend le parfait de tous mes désirs ? Il n'y a et il 
n'y aura jamais fille qui se puisse comparer à elle en beauté, gentillesse, courtoisie et bonne grâce.

(Albert Camus, Les esprits [adaptation de l'œuvre de Pierre de Larivey], 1953)

\subsection{Trois usages de la phrase nominale à ø $\mathrm{N}$}

Selon leur fonctionnement, on distinguera trois types de phrases nominales à ø N .

Type i : Reformulation

Type ii : Présentation d'un concept

Type iii : Jugement

Le premier type se distingue des deux autres par l'information nouvelle/ancienne. Le Type i et le Type iii sont différents en matière d'orientation informationnelle. De plus, à la différence de la classification de il y a ø $\mathrm{N}$, avec les phrases nominales à $\varnothing \mathrm{N}$, chaque type a une forme propre identifiable.

\subsubsection{Type $i$ : Reformulation}

Le premier cas de figure correspond à une structure à 2 termes : thème-rhème. Le thème est le plus souvent caractérisé par une itération, comme diversité, métier et personnalités en (13).

(13) Car ce qui ressort, d'un examen, fût-il superficiel, c'est l'extraordinaire diversité de la création à Paris. Diversité due à des apports successifs et multiples. Cela tient d'abord à la relative marginalité du métier de photographe. Métier alors encore à peine codifié mais en pleine expansion et qui de ce fait attire de fortes personnalités dont l'origine sociale ou nationale aurait freiné l'ascension dans d'autres domaines mieux "balisés". Personnalités souvent riches de talents divers comme le hongrois Brassaï, l'américain Man Ray, l'allemand Bellmer, parfois même extravagants comme Claude Cahun ou le roumain Eli Lotar qui trouvèrent dans la photographie à la fois un moyen de vivre (ou plutôt de survivre), une grande liberté et finalement un moyen de s'accomplir.

(Alain Sayag, Préface de Paris des photographes, 1997)

Les trois $ø \mathrm{~N}$ en tête de phrase nominale reprennent les termes (soulignés) de la phrase précédente. Ils sont thématiques et suivis d'une qualification plus ou moins longue (en italiques), qui reformule l'expression du cotexte antérieur. La cohésion est assurée par la répétition.

On a vu dans la section 3 que le nom nu dans une phrase nominale est le plus souvent abstrait. Mais dans ce type i, le ø N peut être facilement concret comme l'illustre l'exemple suivant. Cette caractéristique diffère du $\varnothing \mathrm{N}$ dans il $y a$, qui accepte difficilement un nom véritablement concret ( $c f$. 5.1.3).

(14) La terre absorbe environ $50 \%$ de l'énergie que lui envoie le soleil. Cette énergie permet à notre planète de se réchauffer. Mais pour qu'elle remplisse effectivement son rôle, il faut l'empêcher de s'échapper. C'est là le rôle dévolu à un "couvercle" constitué de vapeur d'eau et de gaz. Couvercle indispensable qui agit un peu comme une serre pour des plantes : sans cet "effet de serre", la température moyenne de notre planète descendrait à -18 !

(France 2, Dossier, « La conférence de Kyoto », les 12-19/12/1997)

En (14), le nom concret Couvercle (en gras) est une itération de la phrase précédente. La répétition fait de $\varnothing \mathrm{N}$ un objet de discours et un argument de prédication. Le $\varnothing \mathrm{N}$ est donc 
suivi d'un développement rhématique : indispensable qui... lequel constitue le caractère distinctif du type i. La suppression n'en est pas possible. Ainsi, la phrase nominale a une fonction prospective et permet la progression du texte.

Ce type de phrase nominale ressemble à une construction appositive. En effet, au prix de quelques petites modifications typographiques, la conversion en appositive est réalisée comme en (15).

(15) C'est là le rôle dévolu à un "couvercle" constitué de vapeur d'eau et de gaz, couvercle indispensable qui agit un peu comme une serre pour des plantes : sans cet "effet de serre", la température moyenne de notre planète descendrait à -18 !

Sans point, le nom est moins mis en valeur, même si la différence entre (14) et (15) semble minime.

La répétition établit une certaine affinité entre le Type $i$ et le Type I de $i l$ y $a ø N$. En effet, quant à l'orientation informationnelle, le prédicat averbal joue un rôle à la fois prospectif et rétrospectif dans les deux cas : «prospectif» avec la partie rhématique et " rétrospectif » avec le $\varnothing \mathrm{N}$ répété. Mais le contenu informationnel n'est pas identique. Si dans le Type I de il y a ø $N$, le $\varnothing \mathrm{N}$ véhicule une information supposée connue du/des destinataire(s), mais discursivement nouveau (hearer old / discourse new), dans le type i de la phrase nominale, le $\varnothing \mathrm{N}$ représente une information acquise des deux points de vue. Le nom nu du Type i constitue une répétition qui devient en suivant un thème, typiquement connu et ancien.

\subsubsection{Type ii : Présentation d'un concept}

Le second type de phrase nominale à $\varnothing \mathrm{N}$ offre une structure à 1 seul terme. Elle a pour fonction d'introduire un concept. Souvent, un $ø \mathrm{~N}$ constitue à lui seul une phrase, suivie d'une partie complétive indépendante, comme Morale en (16).

(16) Ainsi, il arrive que tel grand patron stagne au niveau d'un directeur financier ou du marketing du secteur privé, âgé de moins de quarante ans. Morale : il est encore plus difficile de trouver le PDG d'une entreprise publique que de rechercher et de sélectionner le PDG d'une entreprise privée.

(Le Monde, 10/07/1991)

Ce type diffère du précédent sur plusieurs points. D’abord, le $\varnothing \mathrm{N}$ apparaît pour la première fois dans le texte. Il est donc aussi nouveau du point de vue du discours que du point de vue des connaissances partagées (discourse new/hearer new). Par suite, sa conversion en apposition n'est pas possible.

En outre, il y a ensuite une partie complétive, le plus souvent sous la forme d'une phrase indépendante qui explique le contenu du ø $\mathrm{N}$ alors que la partie extensive du Type $\mathrm{i}$ qualifie le $\varnothing \mathrm{N}$ à l'intérieur de la même phrase.

De plus, le ø $\mathrm{N}$ du Type ii peut difficilement être concret. Ces noms abstraits sont en fait souvent des « noms généraux », au sens de Halliday et Hasan (1976) et de Legallois (2006), une catégorie de noms appartenant à des catégories lexicales que caractérisent leur faible contenu sémantique et l'ampleur de leur application référentielle. Les travaux de Schmid (2000), qui les nomme Shell nouns, montrent que leur sens est principalement actualisé en contexte, le plus souvent à l'aide de compléments phrastiques ou dans des configurations qui en déterminent l'extension. Comme l'indiquent Halliday et Hasan (1976), les noms généraux sont, en raison de leurs propriétés, fréquemment sollicités dans l'anaphore, voire la cohésion textuelle. Leur capacité à prendre en charge des contenus propositionnels en font des éléments importants de la structuration textuelle. (Schmid 2000 ; Legallois 2008 ; Flowerdew \& Forest 2015). 
Les noms généraux peuvent s'employer de manière anticipée - Huyghe (2018) les désigne comme « advance labels »- en dénotant par avance un conditionneur nominal pour un contenu qui reste à préciser. Observons le mot Résultat en (17).

(17) Les bouchons n'ont (presque) plus de goût

Pour lutter contre le fameux goût de bouchon, ennemi redouté du vin, l'industrie a investi dans la recherche. Résultat: le liège tant décrié regagne du terrain.

(Le Monde, Ophélie Neiman, 17/12/2018)

Le contenu vague de résultat explique que, même sans déterminant, le lecteur attend une spécification qui l'explicite (le liège tant décrié regagne du terrain). Le mot résultat est un contenant qui annonce un contenu ultérieur : l'orientation informationnelle est rétrospective alors que la partie complétive rhématique est prospective. C'est en ce sens que la phrase nominale à $\varnothing \mathrm{N}$ a à la fois une valeur rétrospective et une valeur prospective.

L'exemple (6) constitue une variante de ce type :

(6') Nous avions déjà l'art d'opinion, la démocratie d'opinion ; nous aurons maintenant l'enseignement d'opinion. Il faut renoncer à l'idée que le lycée puisse rester un lieu privilégié de communication du savoir.

Conclusions? Elles sont au nombre de deux. La première était déjà contenue dans mes prémisses.

Conclusions? avec la marque d'interrogation et le caractère porteur du mot incite le lecteur à chercher un coréférent explicite. En effet, la phrase suivante (Elles sont au nombre de deux.) annonce une dualité que le cotexte postérieur (La première était...) complète. Le $ø \mathrm{~N}$ se réfère à un contenu abstrait sans le décrire. Cette caractéristique définitionnelle des noms généraux joue un rôle structurant dans l'organisation textuelle.

Véhiculant peu d'informations sur le référent, les noms généraux, à l'instar des pronoms, fonctionnent comme des éléments substituts afin d'assurer la cohésion.

\subsubsection{Type iii : Jugement}

Le troisième type de phrase nominale à $\varnothing \mathrm{N}$ exprime un jugement ou une appréciation sur ce qui est relaté en amont dans le cotexte. En (18), le mot-phrase Mystère porte un jugement sur la situation énoncée dans la phrase précédente.

(18) Cette nuit, en pleine insomnie, impossible de me rappeler le patronyme d'Isabelle X. Pourquoi mon attention se focalise-t-elle de façon obsessionnelle sur ce détail ? Mystère. Ce nom absent, qu'il me suffirait de chercher sur internet, me démange comme un prurit, il me faut absolument le trouver par moi-même.

(Évelyne Bloch-Dano, Porte de Champerret, 2013)

Le Type iii présente plusieurs traits communs avec le type précédent. Structure à 1 terme, le $\varnothing \mathrm{N}$ peut constituer à lui seul une phrase. Apparaissant pour la première fois dans le texte, il est nouveau du point de vue du discours et du point de vue des connaissances partagées (discourse new/hearer new). La conversion en apposition n'est pas possible.

Ce qui différencie le Type iii du Type ii est avant tout qu'une spécification n'est pas attendue. Le $\varnothing \mathrm{N}$ condense sous forme nominale un contenu propositionnel précédemment exprimé mais le regard est uniquement rétrospectif.

Assez souvent, le contenu du $\varnothing \mathrm{N}$ fait l'objet d'un jugement de valeur. Cet aspect évaluatif est souvent réalisé par la combinaison d'un nom et d'un adjectif axiologique, comme en (19).

(19) Fumer, c'est moche 
Pour tenter de réduire l'attrait de la cigarette, les autorités sanitaires ont voulu effrayer les consommateurs en imprimant des visuels chocs sur les paquets. Peine perdue ?

(Le Monde, 14/05/2015)

En (20), le contenu du ø N Interrogation lui-même fait l'objet d'une évaluation. Ainsi, le nom est-il qualifié par un groupe adjectival (tout à fait gratuite).

(20) Naturellement, je fis ma surprise et je niai. En vérité, je me demandais lequel de ces fourrés avait vu la conjugaison de sœurette et de John Thomas. Interrogation tout à fait gratuite car il n'y avait certainement pas d'écriteau pour l'indiquer, ce pouvait être celui-ci aussi bien que celui-là.

(Raymond Queneau pseud. Sally Mara, Les auvres complètes de Sally Mara, 1962)

L'exemple (2) est une variante de ce type. Malgré la répétition lexicale, la structure textuelle s'avère identique à celle de (20). Le nom nu Guerre sert de support à la prédication adjectivale axiologique injuste, suivi de la conjonction car.

(2') Mais tandis que l'Europe prend ainsi le chemin de la paix, c'est la guerre qui sévit en Asie du sud-est. Guerre injuste car elle résulte en fait de l'intervention armée des États-Unis sur le territoire du Vietnam, guerre détestable puisqu'elle conduit une grande nation en ravager une petite.

Ces phrases nominales à $\varnothing \mathrm{N}$ constituent une prédication extraphrastique portant sur le cotexte gauche. Dans la terminologie de Legallois (2006), c'est une «méta-énonciation ». Le regard reste uniquement rétrospectif.

\subsection{La cohésion et les informations du ø $\mathrm{N}$}

Le tableau 3 récapitule le résultat des observations de cette section.

Tableau 3. Différents usages de il y a ø $\mathrm{N}$ et des phrases nominales à $\varnothing \mathrm{N}$.

\begin{tabular}{|c|c|}
\hline il y $a ø \mathrm{~N}$ & les phrases nominales à $\varnothing \mathrm{N}$ \\
\hline $\begin{array}{l}\text { Type I: Définition } \\
\text { information supposée connue du/des } \\
\text { destinataire(s), mais discursivement } \\
\text { nouvelle } \\
\text { regards prospectif et rétrospectif }\end{array}$ & $\begin{array}{l}\text { Type } i: \text { Reformulation } \\
\text { information ancienne } \\
\text { regards prospectif et rétrospectif }\end{array}$ \\
\hline $\begin{array}{l}\text { Type II : Reconnaissance d'une existence } \\
\text { information nouvelle } \\
\text { regard rétrospectif }\end{array}$ & $\begin{array}{l}\text { Type ii : Présentation d'un concept } \\
\text { information nouvelle } \\
\text { regards prospectif et rétrospectif }\end{array}$ \\
\hline $\begin{array}{l}\text { Type III : Présentation d'un événement } \\
\text { information nouvelle } \\
\text { regard prospectif }\end{array}$ & $\begin{array}{l}\text { Type iii : Jugement } \\
\quad \text { information nouvelle } \\
\quad \text { regard rétrospectif }\end{array}$ \\
\hline
\end{tabular}

Nous constatons que les phrases nominales à $ø \mathrm{~N}$ portent toujours un regard rétrospectif. Ce résultat tient certainement au mécanisme de cohésion exclusivement lexicale de la construction. Soit par répétition (Type i), soit par l'intercession d'une activité mentale (Type ii et Type iii), le $\varnothing \mathrm{N}$ de la phrase nominale doit être relié sémantiquement au cotexte antérieur. Ce qui n'est pas le cas de il y a ø $\mathrm{N}$, dont le nom nu est actualisé en lien avec une tournure spéciale. 


\section{Conclusion}

Par le présent article, nous avons d'abord montré que les noms nus inclus dans il y a et les phrases nominales conservent leur nature substantive. Ensuite, grâce à une analyse quantitative, nous avons identifié les noms nus les plus fréquemment employés dans ces constructions pour montrer quelques différences concernant la nature de ces noms. Puis nous avons identifié les usages typiques de chaque construction: pour il y $a \varnothing \mathrm{N}$ [Définition / Reconnaissance d'une existence / Présentation d'un événement] et pour la phrase nominale à ø $\mathrm{N}$ [Reformulation / Présentation d'un concept / Jugement]. L'examen de l'orientation informationnelle du $\varnothing \mathrm{N}$ nous a permis de distinguer les deux constructions. La phrase nominale, qui introduit un nom nu sans recours à des procédés syntaxiques, a un regard systématiquement rétrospectif.

\section{Notes}

1 Pour un cas d'interchangeabilité, nous citons un exemple de Naganuma (2004). D'après celui-ci, dans le texte suivant, Trahison! (mis en gras) peut se paraphraser par Il y a trahison!

Pour ces cyberéditeurs, l'idée est de revenir aux sources du métier, qui après tout consiste à éditer des textes et non pas à produire du papier encré ! «Il s'agit d'ouvrir un marché complémentaire et aussi d'attirer un public jeune, qui de toute façon fréquente de moins en moins les librairies ", estime Jean-Pierre Arbon, qui reçoit désormais un manuscrit par jour. [alinéa] Trahison ! Après les prêts gratuits en bibliothèque et le "photocopillage », le livre électronique va déstabiliser l'édition noble et enterrer la libraire, s'insurgent une poignée d'éditeurs qui se sont plaints de ces pratiques auprès du ministère de la Culture. (Le Nouvel Observateur, 1775, 12/11/1998)

2 La construction il y a a plusieurs emplois. Il ne semble pas y avoir d'étude systématique sur les différentes catégories en fonction de leurs domaines d'emploi. Quant à son équivalent anglais, la tournure there, Lakoff (1987) propose un classement détaillé. En se fondant sur celui-ci, Togo (2009) propose pour le français la classification suivantes :

a. Phrase définissant un sous-ensemble

Il y a des reptiles qui sont parthénogénétiques.

b. Phrase existentielle locative

Il y a de gros singes dans ce zoo.

c. Phrase événementielle

Qu'y a-t-il donc? -Il y a que j'étouffe.

Il y a grève aujourd 'hui.

d. Phrase existentielle à pseudo-relative

Je voudrais me marier, mais il y a ma mère qui m'en empêche.

e. Phrase existentielle à liste

Qu'est-ce qu'il y a à voir dans cette ville? - Eh bien, il y a le Jardin botanique et la cathédrale.

f. Phrase existentielle dénotant la scène sous les yeux

Regarde! Il y a Jean qui est en train de draguer une fille!

Seules les phrases du groupe c (Phrase événementielle) sont pertinentes pour notre étude. Or, les trois emplois de il y $a ø \mathrm{~N}$ ne se réduisent pas à ce groupe. De ce fait, les trois valeurs que nous identifions (Définition, Reconnaissance d'une existence, Présentation d'un événement) semblent liées aux ø $\mathrm{N}$ et non à il y $a$.

* La présente recherche bénéficie de l'Aide à la recherche scientifique (C) de KAKENHI (20K00621), accordée par la Société Japonaise pour la Promotion des Sciences (JSPS).

\section{Références bibliographiques}

Adler, S. et Legallois, D. (2018). Les noms sous-spécifiés dans le débat parlementaire: analyse fréquentielle et catégorisation modale. Langue française, 198, 19-34.

Van Den Bussche, H. (1988). Typologie des constructions dites appositives. Travaux de linguistique $17,117-135$. 
Chevalier, J.-C. (1969). Exercices portant sur le fonctionnement des présentatifs. Langue française, 1, $82-93$.

Flowerdew, J. et Forest, R. W. (2017). Signalling Nouns in English: A Corpus-Based Discourse Approach. Cambridge : Cambridge University Press.

Grinshpun, Y. (2011). Phrase averbale et presse écrite : le cas des constructions en $[U N+\mathrm{N}+$ expansion). In. Lefeuvre, F. et Behr, I., Les énoncés averbaux autonomes entre grammaire et discours. Paris : Ophrys, 187-203.

Halliday, M. A. K. et Hasan, R. (1976). Cohesion in English. Londres et New York : Longman.

Huyghe, R. (2018). Généralité sémantique et portage propositionnel : le cas de fait. Langue française, 198, 35-50.

Lakoff, G. (1987). Women, fire, and dangerous things: what categories reveal about the mind. Chicago: The University of Chicago press.

Lefeuvre, F. (1999a). La phrase averbale en français. Paris : L'Harmattan.

Lefeuvre, F. (1999b). Les "marqueurs de prédication" dans la phrase averbale en français. Verbum, XXI, 4, 429-438.

Lefeuvre, F. (2005). Le prédicat nominal dans des articles de presse. in Syntaxe et sémantique 2005/1 ( ${ }^{\circ} 6$ ), 181-198< <https://www.cairn.info/revue-syntaxe-et-semantique-2005-1-page-181.htm>.

Legallois, D. (2006). Quand le texte signale sa structure: la fonction textuelle des noms sousspécifiés. Corela HS-5, 1-24.

Legallois, D. \& V. Lenepveu (2014). L'évaluation dans les textes: des relations interpropositionnelles aux séquences discursives. Langue française, 184, 17-33.

Naganuma, K. (2004). Furansugo ni okeru yûhyô no meishi gentei no bunpô. Tokyo: Sobishuppansha.

Picabia L. (1983). Remarques sur le déterminant zéro dans les séquences en il y a. Le français moderne, 51(2), 157-171.

Picabia, L. (1986). Il y a démonstration et démonstration: réflexion sur l'article zéro. Langue française, 72, 80-101.

Picabia, L. (1987). Quand y a-t-il générique ? in Kleiber, G. (éd) Recherches linguistiques XII. Paris : Klincksieck, 235-260.

Picabia, L. (1991). Article zéro et structures apposées. Langage, 102, 88-102.

Prince, E. F., (1981). Toward a Taxonomy of Given/New Information. in Cole P. (ed.), Radical Pragmatics, New York, Academic Press, 223-254.

Prince, E. F., (1992). The ZPG Letter: Subjects, Definiteness, and Information-Status, in Thompson, S. et Mann, W. (eds), Discourse Description: Diverse Analyses of a Fund Raising Text, Philadelphia : John Benjamins, 295-325.

Riegel, M., Pellat, J.-C. et Rioul, R. (2018). Grammaire méthodique du français. Paris : Presses Universitaires de France.

Schmid, H.-J. (2000). English abstract nouns as conceptual shells. Berlin et New York: Mouton de Gruyter.

Takagaki, Y. (2018). La cohésion lexicale en phrase nominale avec un nom sans déterminant. Actes $d u \quad C M L F \quad 2018-\sigma^{e}$ Congrès mondial de linguistique française. $<$ https://doi.org/10.1051/shsconf/20184606012>.

Togo, Y. (2011). Arawasu Bunpô, Tokyo : Hakusuisha. 